\title{
Draft Genome Sequences of Two Phytoplasma Strains Associated with Sugarcane Grassy Shoot (SCGS) and Bermuda Grass White Leaf (BGWL) Diseases
}

\author{
Kiran Kirdat, ${ }^{1,2}$ Bhavesh Tiwarekar, ${ }^{1}$ Vipool Thorat, ${ }^{1}$ Nitin Narawade, ${ }^{1}$ Dhiraj Dhotre, ${ }^{1}$ Shivaji \\ Sathe, ${ }^{2}$ Yogesh Shouche, ${ }^{1}$ and Amit Yadav ${ }^{1, \dagger}$ \\ ${ }^{1}$ National Centre for Microbial Resource, National Centre for Cell Science, Ganeshkhind, Pune 411007 , \\ India \\ ${ }^{2}$ Department of Microbiology, Tuljaram Chaturchand College, Baramati, Maharashtra 413102, India
}

\section{Abstract}

We performed whole-genome sequencing of two phytoplasmas associated with sugarcane grassy shoot (SCGS) and Bermuda grass white leaf diseases. These are the first draft genomes of SCGS phytoplasma (strain SCGS) and 'Candidatus Phytoplasma cynodontis' (strain LW01) and may help to delineate these phytoplasmas at a finer taxonomic level.

\section{Genome Announcement}

'Candidatus Phytoplasma' is a cell wall-less, not-yet-cultivated, obligate bacterial pathogen associated with diseases of hundreds of plant and crop species (Rao et al. 2017). sugarcane grassy shoot (SCGS) phytoplasma causes severe proliferation of tillers leading to the typical grassy appearance of sugarcane (Saccharum officinarum L.), hence the name 'grassy shoot' (Nasare et al. 2007; Yadav et al. 2017). The SCGS disease contributes to losses of 5 to $20 \%$ in the plant crop of sugarcane and these losses are higher in the ratoon crop (Yadav et al. 2013). The Bermuda grass white leaf (BGWL) phytoplasma causes 'white leaf' disease in Cynodon dactylon (L.) Pers. and has been described as 'Candidatus Phytoplasma cynodontis' (Marcone et al. 2004). A sugarcane plant sample (strain SCGS) exhibiting grassy shoot symptoms and a Bermudagrass sample (strain LW01) showing white leaf symptoms were collected from Pune, Maharashtra, India. The presence of phytoplasmas was confirmed by detection and sequencing of the 16S ribosomal RNA (rRNA) gene amplicons from SCGS (MN889545) and LW01 (LT558776) samples. These phytoplasma strains belonged to the 16SrXI and 16SrXIV groups, respectively, according to the restriction fragment length polymorphism patterns of their 16S rRNA gene sequences, which were digested in silico with selected restriction endonuclease enzymes, using online tool IPhyClassifier (Zhao et al. 2009).

Total nucleic acids were extracted from the SCGS and LW01 samples and were enriched for prokaryotic DNA selection using the NEBNext microbiome enrichment kit (New England BioLabs). The enriched DNA of both samples was amplified using illustra Ready-To-Go GenomiPhi V3 DNA amplification kits and was sequenced on the Illumina HiSeq platform. Simultaneously, the enriched DNA of SCGS and LW01 were sequenced on the Oxford Nanopore Technology (ONT) platform.

Sequencing of the SCGS and LW01 samples resulted in 22,765,620 reads $(150 \times 2$ chemistry) and $22,630,326$ reads $(150 \times 2$ chemistry), respectively, using the Illumina HiSeq

${ }^{\dagger}$ Corresponding author: A. Yadav; amityadav@nccs.res.in

The author(s) declare no conflict of interest.

Accepted for publication 8 February 2020.

\section{Funding}

The work was funded by the Science and Engineering Research Board (SERB), Department of Science and Technology, Government of India, grant number SERB/2016/000752.

Keyword

phytoplasmas 
platform. All Illumina reads were quality checked with FastQC v0.11.8 (Brown et al. 2017). The ONT sequencing data were base-called with quality filtering (>Q7) using GUPPY, which generated 50,541 reads for SCGS and 41,237 for LW01. All QC-passed Illumina reads (>Q30) were subjected to metagenomic assembly using MEGAHIT v1.1.3 (Li et al. 2016). This assembly was subjected to binning, using MetaBAT2 v2.12.1 (Kang et al. 2015). Raw reads were mapped on bins corresponding to phytoplasma. Additionally, Illumina reads were mapped on all available phytoplasma genome sequences, using Bowtie2 v2.2.6 (Langmead and Salzberg 2012). Finally, all mapped reads and QC-passed ONT reads were used to generate the hybrid assembly in SPAdes v3.11.1 (Bankevich et al. 2012) and Unicycler v0.4.8 (Wick et al. 2017). Bin quality check, genome completeness, and taxonomic assignments were performed using CheckM v1.0.14 (Parks et al. 2015). Assembly quality was checked using QUAST v5.0.2 (Gurevich et al. 2013). The genome coverage was calculated using BBMap (Bushnell, 2014). All obtained scaffolds were used as the queries to run a BLASTX search against the National Center for Biotechnology Information (NCBI) nonredundant protein database, using Blast2GO to identify contaminating scaffolds. Both genomes were annotated using the NCBI Prokaryotic Genome Annotation Pipeline (Zhao et al. 2012).

The final SCGS assembly contained 29 scaffolds corresponding to $505,173 \mathrm{bp}$, including a putative plasmid of $2,976 \mathrm{bp}$. This genome was found to be $95.43 \%$ complete with $333.98 \times$ coverage and the GC content was $19.86 \%$. The SCGS genome was predicted to have 404 protein-coding genes, 12 transfer RNA (tRNA), and two rRNA genes. Similarly, the final LW01 assembly contained 21 scaffolds corresponding to $483,935 \mathrm{bp}$. This genome was found to be $91.32 \%$ complete with $373.6 \times$ coverage and the GC content was $20.46 \%$. The LW01 genome was predicted to have 425 protein-coding genes, 13 tRNA, and three rRNA genes. The 16S rRNA gene sequences of SCGS and LW01, obtained from their respective assemblies, showed 98.07 and $99.59 \%$ similarity, respectively, with the reference sequence of 'Candidatus Phytoplasma cynodontis' strain BGWL-C1, when analyzed on EzBioCloud Database to find out their closest taxonomic relative (Yoon et al. 2017).

\section{Data Availability}

The Whole Genome Shotgun sequence for strains SCGS and LW01 have been deposited in the DDBJ/ENA/GenBank database under accession numbers VWXM00000000 and VWOH00000000, respectively. The version described in this paper is VWXM01000000 and VWOH01000000. The sequence of the SCGS plasmid (pSCGS) is available under accession number NZ_CM018785.1

\section{Acknowledgment}

The authors thank K. Jangid (NCMR-NCCS, Pune) for his contributions to the improvement of this manuscript.

\section{Literature Cited}

Bankevich, A., Nurk, S., Antipov, D., Gurevich, A. A., Dvorkin, M., Kulikov, A. S., Lesin, V. M., Nikolenko, S. I., Pham, S., Prjibelski, A. D., Pyshkin, A. V., Sirotkin, A. V., Vyahhi, N., Tesler, G., Alekseyev, M. A., and Pevzner, P. A. 2012. SPAdes: A new genome assembly algorithm and its applications to single-cell sequencing. J. Comput. Biol. 19:455-477.

Brown, J., Pirrung, M., and McCue, L. A. 2017. FQC dashboard: integrates FastQC results into a web-based, interactive, and extensible FASTQ quality control tool. Bioinformatics 33:3137-3139.

Bushnell, B. 2014. BBMap: A fast, accurate, splice-aware aligner. Lawrence Berkeley National Lab, Berkeley, CA, U.S.A..

Gurevich, A., Saveliev, V., Vyahhi, N., and Tesler, G. 2013. QUAST: Quality assessment tool for genome assemblies. Bioinformatics 29:1072-1075.

Kang, D. D., Froula, J., Egan, R., and Wang, Z. 2015. MetaBAT, an efficient tool for accurately reconstructing single genomes from complex microbial communities. PeerJ 3:e1165.

Langmead, B., and Salzberg, S. L. 2012. Fast gapped-read alignment with Bowtie 2. Nat. Methods 9:357-359.
Li, D., Luo, R., Liu, C.-M., Leung, C.-M., Ting, H.-F., Sadakane, K., Yamashita, H., and Lam, T.W. 2016. MEGAHIT v1.0: A fast and scalable metagenome assembler driven by advanced methodologies and community practices. Methods 102:3-11.

Marcone, C., Schneider, B., and Seemüller, E. 2004. 'Candidatus Phytoplasma cynodontis', the phytoplasma associated with Bermuda grass white leaf disease. Int. J. Syst. Evol. Microbiol. 54:1077-1082.

Nasare, K., Yadav, A., Singh, A. K., Shivasharanappa, K. B., Nerkar, Y. S., and Reddy, V. S. 2007. Molecular and symptom analysis reveal the presence of new phytoplasmas associated with sugarcane grassy shoot disease in India. Plant Dis. 91:1413-1418.

Parks, D. H., Imelfort, M., Skennerton, C. T., Hugenholtz, P., and Tyson, G. W. 2015. CheckM: Assessing the quality of microbial genomes recovered from isolates, single cells, and metagenomes. Genome Res. 25:1043-1055.

Rao, G., Madhupriya, V. T., Manimekalai, R., Tiwari, A., and Yadav, A. 2017. A century progress of research on phytoplasma diseases in India. Phytopathogenic Mollicutes. 7:1-38.

Wick, R. R., Judd, L. M., Gorrie, C. L., and Holt, K. E. 2017. Unicycler: Resolving bacterial genome assemblies from short and long sequencing reads. PLOS Comput. Biol. 13:e1005595. 
Yadav, A., Deokule, S., and Prasad, D. T. 2013. Detection, diversity and differential expression studies of Sugarcane Grassy Shoot (SCGS) phytoplasma. Ph. D. thesis. University of Pune, India., http://hdl.handle.net/10603/106870

Yadav, A., Thorat, V., Deokule, S., Shouche, Y., and Prasad, D. T. 2017. New subgroup 16SrXI-F phytoplasma strain associated with sugarcane grassy shoot (SCGS) disease in India. Int. J. Syst. Evol. Microbiol. 67:374-378.

Yoon, S.-H., Ha, S.-M., Kwon, S., Lim, J., Kim, Y., Seo, H., and Chun, J. 2017. Introducing EzBioCloud: A Taxonomically united database of 16S rRNA gene sequences and whole-genome assemblies. Int. J. Syst. Evol. Microbiol. 67: 1613-1617.

Zhao, Y., Wei, W., Lee, I. M., Shao, J., Suo, X., and Davis, R. E. 2009. Construction of an interactive online phytoplasma classification tool, iPhyClassifier, and its application in analysis of the peach X-disease phytoplasma group (16SrllI). Int. J. Syst. Evol. Microbiol. 59:2582-2593.

Zhao, Y., Wu, J., Yang, J., Sun, S., Xiao, J., and Yu, J. 2012. PGAP: Pan-genomes analysis pipeline. Bioinformatics 28:416-418. 